\title{
Could proton pump inhibitors be re-purposed against SARS-CoV-2? - A hypothesis based on existing evidences
}

\author{
Avik Ray ${ }^{1}$, Swati Sharma ${ }^{1}$, and Balakrishnan Sadasivam ${ }^{1}$ \\ ${ }^{1}$ All India Institute of Medical Sciences Bhopal
}

May 29, 2020

\begin{abstract}
Proton pump inhibitors (PPIs) belong to a well-known class of pharmaceutical agents which target the gastric epithelial $\mathrm{H}+/ \mathrm{K}+$ ATPase. Although the major therapeutic uses of the PPIs are in gastric-acid related diseases, evidences are suggestive of a pleiotropic nature of the compounds. They have been used for the treatment of conditions such as eosinophilic esophagitis and Helicobacter pylori infection, in addition to their conventional use in gastro-oesophageal reflux disease and peptic ulcers. The current pandemic of COVID-19 has put forward a challenge to find treatment options. While multiple studies have been conducted, and many are currently on-going, we have not been able to come up with any specific agent against SARS-CoV-2. PPIs have shown antiviral potencies in various in vivo and in vitro studies. Results from these studies point toward a promising role of PPIs for infections caused by Marburg virus, Influenza virus and Rhinovirus to mention a few. However, neither have they been considered for any clinical trials nor have any guidelines suggested their re-purposing against SARS-CoV-2 till date. In this review, we discuss the probable pathways and cellular machineries via which PPIs could show beneficial therapeutic effects against the novel coronavirus based on the existing evidences.
\end{abstract}

\section{Abbreviations}

ACE2, angiotensin-converting enzyme 2; ATP, adenosine triphosphate; CDC, The Centers for Disease Control and Prevention; COPD, chronic obstructive pulmonary disease; COVID-19, Coronavirus disease 2019; ESCRT, endosomal sorting complex required for transport; FDA, Food and Drug Administration; GERD, Gastroesophageal reflux disease; ICAM-1, Intercellular Adhesion Molecule 1; IL, Interleukin; MERS-CoV, Middle East respiratory syndrome coronavirus; mRNA, messenger RNA; OR, odds ratio; P-CAB, potassiumcompetitive acid blocker; PPI, proton pump inhibitors; R\&D, Research and Development; RV, Rhinovirus; SARS-CoV, Severe acute respiratory syndrome coronavirus; SARS-CoV-2, Severe acute respiratory syndrome coronavirus 2; TNF, tumor necrosis factor; v-ATPase, vacuolar ATPase; WHO, World Health Organization

\section{Introduction}

Proton pump inhibitors (PPIs) are one of the most widely prescribed group of drugs for the treatment of various gastric acid related disorders such as peptic ulcer disease and gastro-oesophageal reflux disease (GERD). Introduced in 1989 with the discovery of omeprazole, the therapeutic class has been widening ever since, with agents like esomeprazole, lansoprazole, pantoprazole, rabeprazole and dexlansoprazole. Vonoprazan, a novel potassium-competitive acid blocker (P-CAB) which has been approved in Japan, provides reversible suppression of the gastric acid by inhibiting $\mathrm{K}^{+}$from binding to the gastric $\mathrm{H}+/ \mathrm{K}+$-ATPase pumps (Yang et al., 2018). The high potency of all of these agents to increase the intragastric $\mathrm{pH}$ along with a favourable adverse effect profile have made them a popular choice among physicians.

PPIs have shown to have multiple therapeutic effects besides on gastric acid diseases (Yu et al., 2017). One factor which might contribute to the pleiotropic nature of PPIs is the presence of benzimidazole scaffold in 
their structure, which is considered a privilege since it imparts the ability to simultaneously target various biological molecules (Kaur, Kaur, \& Silakari, 2014).

Considering the current pandemic of COVID-19 and the necessity to find a drug for its treatment, it is of utmost importance that the antiviral potencies of the already approved drugs be explored in order to consider them for re-purposing. Since SARS-CoV-2 shows a high degree of phylogenetic similarities with previously identified coronaviruses such as SARS-CoV and MERS-CoV (Lu et al., 2020), it might be a possibility that drugs which show effects on these viruses may be potential candidates for screening against the novel coronavirus.

\section{General antiviral properties of PPIs}

Currently, there are very few antiviral agents available for the treatment for viral infections. Global pandemics of viral infections such as the ongoing one of COVID-19 point towards the need for us to expand the armoury. Conventionally used antiviral drugs such as zidovudine, acyclovir and foscarnet have shortcomings in terms of limited applications, serious adverse reactions and development of resistance. A patent registered in as early as 1999 showed glimpses of a potential future role of PPIs as antiviral agents by demonstrating their ability to inhibit viral serine protease (PubChem, 1999).

We hereby review the evidences of PPIs suggestive of their potential actions against SARS-CoV-2 and explore the possibility of a hypothesis of their therapeutic roles in the management of COVID-19.

\section{Methodology and literature search strategy}

We conducted a thorough literature search using different electronic databases (PubMed, Google Scholar, Embase and Science Direct) up to May 15 2020. The search was performed using different search terms alone, or in any combinations such as "SARS-CoV-2", "COVID-19", "SARS-CoV-2 disease", "novel coronavirus", "clinical trial", "proton pump inhibitors", "PPIs", "antiviral", "omeprazole", "pantoprazole", "lansoprazole", "PPI", "treatment", "dexlansoprazole", "esomeprazole", "virus", "viral entry", "infection", "entry inhibitor", "viral replication", "in vitro", "H+ $\mathrm{K}+$ ATPase", "in vivo", "ACE2", "gastric epithelium", "ARDS", "pneumonia", "repurposing". Only articles in English with clearly mentioned information were included.

\section{As an anti-inflammatory and anti-fibrotic agent}

PPIs might be associated with anti-inflammatory and antioxidative actions (Becker et al., 2006; Sasaki, Nakayama, Yasuda, \& Yamaya, 2011; Vanfleteren, Spruit, Wouters, \& Franssen, 2016). An in vitrostudy has shown that they can inhibit the production of pro-inflammatory cytokines such as IL-6, IL-8 and TNF- $\alpha$ (Sasaki, Nakayama, Yasuda, \& Yamaya, 2011). Further, there are evidences to support protective role of omeprazole and lansoprazole in human gastric epithelial and endothelial cells against oxidative stress (Becker et al., 2006). Lansoprazole has been shown to reduce the number of monocytes expressing ICAM-1 in the peripheral blood (Ohara \& Arakawa, 1999). Additionally, omeprazole can reduce cytokine production in duodenal epithelial cells as was observed in an in vivo study (Kountouras, Boura, \& Lygidakis, 2000). They have also been associated with antiproliferative and antifibrotic properties (Ghebre \& Raghu, 2016). As cases of cytokine storm in COVID-19 have been reported (Ye, Wang, \& Mao, 2020) and pirfenidone, an antifibrotic drug, has been considered for a phase 3 trial against SARS-CoV-2 ( Clinicaltrial.gov, 2020), it would be interesting to explore whether PPIs can attenuate the release and actions of the cytokines.

\section{Action on vacuolar ATPase and impact on $\mathrm{pH}$}

Host-factors essential for viral replication have been identified through various genome-wide screening studies. Host-targeted strategies are less susceptible to mutations of the viral strains. One of such host factors are vacuolar ATPase (v-ATPase).

Located on the plasma membrane and on the surface of acidic organelles such as lysosomes and endosomes, v-ATPase is one of the key factors controlling cellular pH (De Milito \& Fais, 2007). It is a vacuolar-type proton pump which maintains the acidic $\mathrm{pH}$ inside endosomes and lysosomes. They are present on the 
membranes of acidic intracellular organelles such as secretory granules, endosomes, lysosomes and transGolgi network and act as the major proton-extruding pump. v-ATPase consists of a number of subunits with two functional domains, $V_{0}$ (the integral domain) and $V_{1}$ (the peripheral domain) (Nelson, 1991). The G1 subunit, a part of $\mathrm{V}_{1}$, is associated with ATP hydrolysis. Its activity could be inhibited by the PPIs which can hence, regulate the intracellular pH (Udelnow et al., 2011).

Most of the enveloped viruses utilizes the endocytic pathway prior to their fusion with the membranes of the host cell organelles such as endosomes and lysosomes. A key requirement for the normal endocytic pathway functioning is to have an acidic internal $\mathrm{pH}$. The viruses utilize $\mathrm{pH}$ sensing as an evolutionary strategy to monitor the endosomal maturity. Two major sensing strategies used by the viruses for induction of membrane fusion are direct sensing of the $\mathrm{pH}$ leading to conformational changes in the envelope glycoproteins and protease mediated proteolytic cleavage (Mazzon \& Marsh, 2019). Increase in this $\mathrm{pH}$ can hence lead to inhibition of viral fusion and replication. Besides, modulation of $\mathrm{pH}$ is important for exocytosis as well, leading to viral dissemination (Marshansky \& Futai, 2008).

v-ATPase mediated acidification of endosome is an important step for entry of several viruses including coronaviruses (Belouzard, Millet, Licitra, \& Whittaker, 2012). A 3C-like protease (3CL ${ }^{\text {pro }}$ ) produced by SARS-CoV plays an important role in viral replication (Ziebuhr, Snijder, \& Gorbalenya, 2000). A study has shown that 3CL $\mathrm{CL}^{\text {pro }}$ binds to and cleaves v-ATPase G1 subunit, thus disrupting its function (Lin et al., 2005). Although several v-ATPase inhibitors have shown antiviral effects, they have not been approved yet, mainly due to their toxicity concerns. Additionally, they are poorly soluble in water, leading on to drug delivery challenges. One such example is diphyllin which can effectively inhibit infection by influenza virus (Chen et al., 2013) and feline coronavirus (Cj, Chang, Fang, Cheng, \& Wang, 2018) among others.

An in-vitro screening of 60 FDA approved drugs performed by Touret et al. 2020 revealed anti-viral potency of omeprazole, supporting its repurposing against COVID-19 (not yet published). Omeprazole, along with Vonoprazan, was proved to be associated with increase in $\mathrm{pH}$ inside endosomes and golgi apparatus. The postulated hypotheses are that it happens either by blocking v-ATPase pump or by acting as a pH buffer. Such modifications of $\mathrm{pH}$ would interfere with the processing of the spike (S1) protein by the endosomal proteases and restrict the egress of SARS-CoV-2.

SARS-CoV-2, like SARS-CoV, enters into the cells by binding of its S1 protein to the surface ACE2 receptors. SARS-CoV enters into an acidic environment inside the endosomes where proteolysis occurs, leading to release of viral genome into the cellular cytoplasm. Lysosomes, a key organelle of the endocytic pathway, also relies on v-ATPase for its acidic internal $\mathrm{pH}$. There is an extension of the endocytic pathway in the form of autophagosomes forming complexes with lysosomes, leading to the release of the viral genome for SARS-CoV (Prentice, McAuliffe, Lu, Subbarao, \& Denison, 2004). Since SARS-CoV-2 binds to ACE2 receptor similar to SARS-CoV and is also affected by lysosomotropic agents such as hydroxychloroquine, which can increase the endosomal $\mathrm{pH}$ and inhibit viral replication, it might be a possibility that this novel coronavirus also utilizes the endocytic machinery for entering into the target cell. Hydroxychloroquine has been tried against SARS-CoV-2, although without much success (Geleris et al., 2020). However, such agents have shown activity against SARS-CoV (Simmons, Gosalia, Rennekamp, Reeves, Diamond, \& Bates, 2005). Hence it might be a fair shot to explore whether PPIs, which cause similar alteration of endosomal $\mathrm{pH}$, can be targeted against SARS-CoV-2.

The concept of lysosomotropism was first mentioned in a paper where the possible antiviral properties of these agents was emphasized (de Duve, de Barsy, Poole, Trouet, Tulkens, \& Van Hoof, 1974). Most of the weak bases having lipophilic properties are thought to show lysosomotropism to some extent. This allows these agents to passively travel through the endosomal membrane and get trapped inside the lumen of the endosomes following protonation-based trapping.

Quite interestingly, a study has shown that in cells treated with lysosomotropic agents such as $\mathrm{NH}_{4} \mathrm{Cl}$ or Bafilomycin A1 (inhibitor of endosomal/lysosomal v-ATPase), ACE2 receptors present on the cell surface get trapped within perinuclear vacuoles, suggesting that function of ACE2 might be inhibited by these agents 
and hence, prevent the entry of SARS-CoV-2 into the host cell (Wang et al., 2008).

Although high concentrations of Bafilomycin affect maturation of endosomes, at low concentration it acts as a selective v-ATPase blocker and raises the $\mathrm{pH}$ without hampering the endosomal trafficking, supporting the fact that blocking the proton pumps by PPI would not alter the normal endosomal functioning (Bayer, Schober, Prchla, Murphy, Blaas, \& Fuchs, 1998).

Omeprazole can block the function of v-ATPase in renal cortical cells and medullary endosomes as seen in a study on rats (Sabolić, Brown, Verbavatz, \& Kleinman, 1994). Evidences also point towards a potential activity of lansoprazole as a v-ATPase inhibitor in gastric mucosa (Sachs, Prinz, Loo, Bamberg, Besancon, \& Shin, 1994). Omeprazole has also been reported to have anti-malarial property owing to its ability to inhibit v-ATPase in the plasma membrane of Plasmodium falciparum (Saliba \& Kirk, 1999). It inhibits intracellular v-ATPase at a concentration much higher than what is needed to target gastric $\mathrm{H}+/ \mathrm{K}+$-ATPase. On similar lines, both omeprazole and esomeprazole have been proposed to have roles in cancer therapy by increasing the intracellular $\mathrm{pH}$, thus improving the sensitivity to cytotoxic agents (De Milito \& Fais, 2005). More recently, reports are suggestive of these two agents capable of altering the localisation of the v-ATPase inside the cells as well (Brecher, Schornberg, Delos, Fusco, Saphire, \& White, 2012). This strengthens the claim about the antiviral potency of PPIs and the need for further studies regarding their possible re-purposing for COVID-19.

If effectiveness of PPIs against SARS-CoV-2 gets proven, drug delivery through nanoparticles could also be thought of, leveraging the virus-like dimensions and in vivo biodistribution of nanocarriers, enhancing their antiviral potency.

While we discuss about the potential role of PPI against SARS-CoV-2 by blocking the v-ATPase pump, one point which should be considered is that the normal physiological endosomal trafficking should not be hampered. Further, infectivity of certain viruses using alternative strategies might increase due to inhibition of the endosomal pathway. Hence, in light of non-availability of an alternate therapeutic option, the focus should be laid on short-term inhibition of the endocytic pathway by the lysosomotropic agents.

\section{Targeting ESCRT}

Many of the enveloped viruses utilize a host factor, the endosomal sorting complex required for transport (ESCRT), for their progression from an infected cell. Coronaviruses encode classical motifs which interact with ESCRT, one such example being P(T/S)AP (Sobhy, 2016). This motif engages a protein Tsg101 in ESCRT-I, leading to delivery of ESCRT-III to viral budding sites, causing release of the virus particles by membrane scission (Votteler, \& Sundquist, 2013). A study showed that tenatoprazole and esomeprazole, PPIs which are prodrugs, target a cysteine residue (C73) in the N-terminal domain of Tsg101, disrupting its binding with ubiquitin and preventing its localization to the plasma membrane budding site, thus inhibiting viral replication (Watanabe et al., 2020). For this, they required conversion to their active form inside the cells. Tsg101 and ESCRT have been found to help in replication of Ebola virus (Tabata et al., 2016) and Dengue virus (Irie, Licata, McGettigan, Schnell, \& Harty, 2004) besides a few others (Chua et al., 2007; Strickland et al., 2017). While this demonstrates the importance of CoV envelop (E)-host proteinprotein interactions, we do not have enough information about such virus-host factor interactions yet and hence, we cannot say conclusively whether the viral nucleocapsid envelope causes membrane scission in an ESCRT-dependent manner or not. It is essential to find this out since PPIs could be re-purposed against SARS-CoV-2 based on this mechanism of action. Like tenatoprazole and esomeprazole, other PPIs in the market also target C73 in Tsg101 (Watanabe et al., 2020), leading to a hypothesis that prazole compounds could be effective against a broad spectrum of viruses by inhibiting replication, including SARS-Cov-2.

\section{Learnings from COPD}

Patients who experience frequent exacerbations of chronic obstructive pulmonary disease (COPD) show high susceptibility to viral infections and poor ability to prevent their replication (George et al., 2014). PPIs have shown to have impact on such viral infections (herpes virus, rhinovirus), displaying potential to 
prevent COPD exacerbations (Long et al., 2015). A randomized trial showed that lansoprazole use could be associated with reduction of frequency of common cold and COPD exacerbation, thus attenuating chances of contracting viral infections (Sasaki et al., 2009).

GERD is often found to be associated with COPD as a comorbidity, with its prevalence being higher in COPD patients as compared to healthy controls (Casanova et al., 2004). It often leads to frequent COPD exacerbation in patients. PPIs are the first-line drug of choice for managing GERD. Although there are mixed evidences, a recent nationwide study with 3,485 patients of COPD having symptomatic GERD showed that PPI use is associated with a better outcome in terms of risk of acute exacerbation of COPD and mortality (Su et al., 2018). By doing so, it might indirectly lead to protection of such patients against viral infections.

\subsection{Rhinovirus}

Rhinoviruses (RV) are commonly associated with COPD exacerbations. The viral mRNA enters into the acidic endosomes, leading to increased expression of intercellular adhesion molecule-1 (ICAM-1) (Yu et al., 2017). ICAM-1 is the major rhinovirus receptor located on respiratory epithelial cells similar to ACE2 for SARS-CoV-2 (George et al., 2014). Lansoprazole and omeprazole have been reported to supress the expression of ICAM-1 mRNA probably due to the inhibition of v-ATPase, leading to increase of the endosomal pH (Sasaki et al., 2005). It would hence be useful to find out if similar effects of PPIs on SARS-CoV-2 could be observed.

\section{Actions on ACE2}

PPIs modulate both gastric and small intestinal acidity. It is evident that they exert their direct pH lowering effect on stomach and duodenum, and not beyond that in the gastrointestinal tract (Watanabe, Suzuki, \& Suzuki, 1990).

CDC has recently included diarrhoea as one of the non-respiratory symptoms of COVID-19 (CDC, 2020). SARS-CoV-2 uses ACE2 as its receptor for entry into human body. The glandular cells of gastrointestinal epithelium, mainly of the stomach, duodenum and rectum, express ACE2 protein and have been shown to exhibit the SARS-CoV-2 viral nucleocapsid protein in patients with COVID-19 (Xiao, Tang, Zheng, Liu, $\mathrm{Li}$, \& Shan, 2020). ACE2 has also been shown to play an important role in amino acid transport in the intestines and the composition of the gut microbiota. It is also involved in intestinal inflammation and diarrhoea (Patten, \& Abeywardena, 2017). This delineates the presence of the virus in the gastrointestinal tract of humans and could account for the diarrhoea associated with the disease. The effect of PPI use on ACE2 activity and subsequently on the entry of SARS-CoV-2 in human body would be worth noting.

There exists a $\mathrm{H}^{+} / \mathrm{K}^{+}$ATPase pump in colon (cHK- $\alpha$ ) similar to its homolog in the stomach (gHK- $\alpha$ ) as shown by studies on rabbit and guinea pig. Omeprazole reduces the proton $\left(\mathrm{H}^{+}\right)$secretory activity of this pump as well, although this reduction is not as much, leading to decreased release of $\mathrm{H}^{+}$in the large intestine and hence causing increased $\mathrm{pH}$ in the lumen (Watanabe et al., 1990).

ACE2 enzyme activity varies with $\mathrm{pH}$. A pH of 7.5 is said to be optimal for its functioning (Aragão et al., 2011). As discussed, PPIs tend to acidify the intracellular environment by inhibiting v-ATPase. Hence, the ACE2 would be rendered dysfunctional or at least less functional (Lee et al., 2015), thereby hindering the entry of SARS-CoV-2 into the cell.

ACE2 is a zinc metalloprotease and chronic PPI use is known to reduce zinc levels in the body by depleting zinc stores as well as by preventing absorption of supplemental zinc (Farrell et al., 2011). The dose of PPI required to exert anti-SARS-CoV-2 effect has been estimated by Bojkova et al. (2020) to be very high (not yet published). Their use at such high levels could lead to zinc deficiency despite being used for shorter duration. Serum ACE activity in rats and guinea pigs has been shown to be affected adversely by zinc deficiency (Reeves, \& O'Dell, 1986). ACE2 exhibits substantial structural homology to ACE and is in fact also known by the name ACEH (ACE homolog) (Turner, Tipnis, Guy, Rice, \& Hooper, 2002). It would be interesting to see the effect of use of PPIs on ACE2 pertaining to zinc levels. 
Additionally, the $\mathrm{pH}$ increasing effect of the PPIs would make the environment inside the gastric and intestinal lumen slightly basic, leading to damage of the fatty viral membrane (D et al., 2020), thus disrupting its binding to the host cell receptor and ultimately leading to its death. This is one more additional factor for considering exploration of the potential roles of PPIs against SARS-CoV-2.

\section{Role as an add-on}

In addition to the direct antiviral effects, PPIs might also be used as an add-on to certain therapeutic agents. In an in-silico study by Bojkova et al. 2020, omeprazole increased the efficacy of aprotinin, a serine protease inhibitor, and remdesivir, a potential therapeutic agent for SARS-CoV-2, currently under clinical investigation, by 2.7 -fold and 10-fold respectively (not yet published). Thus, combination of aprotinin and remdesivir with omeprazole might be a potential therapy candidate for the treatment of COVID-19.

\section{Clinical evidences}

A propensity score matched retrospective cohort-based study, which tried to identify the role of famotidine in improving the clinical outcomes of hospitalized COVID-19 patients, showed, as a secondary finding, that proton pump inhibitors do not impart any clinical benefit in terms of reduction of deaths or need for intubation (Freedberg et al., 2020). However, a case series of three patients reported from China has mentioned two cases of COVID-19 who were on intravenous esomeprazole (40 mg twice daily) while they were hospitalized and were subsequently discharged. The first case was that of a 32-year-old male who had prolonged diarrhoea and fatigue before developing cough and breathing difficulties. The second case was of an 82-years-old asymptomatic female who had a contact history with a laboratory-confirmed COVID19 patient. Although there was a definite correlation between proton pump inhibitor administration and favourable clinical outcome, several concomitant medications such recombinant interferon and umifenovir could have confounded the outcomes in both the cases, denying any causal relationship between the two (Liu et al., 2020). It is worth mentioning that in a retrospective case-control study by Blanc et al. 2020 on 179 elderly patients, those on PPIs were 2.3 times less likely (Odds Ratio $[\mathrm{OR}]=0.4381,95 \%$ confidence interval [0.2331 to 0.8175], $\mathrm{p}=0.0053)$ to be infected by SARS-CoV-2 and develop COVID-19 as compared to those not taking PPIs (not yet published). Hence clinical benefits of PPIs should really be explored through larger, appropriately powered studies. In fact, an R\&D blueprint for experimental treatments, which has been developed by the World Health Organization (WHO) in order to join hands globally and accelerate the process of finding therapeutics against SARS-CoV-2, mentions the probable roles of omeprazole, lansoprazole and rabeprazole, either alone or in combinations with other drugs, for the treatment of COVID-19 (WHO, 2020).

\section{Conclusions}

All of the evidences, as discussed, point toward a potential role of PPIs as antiviral agents against SARS$\mathrm{CoV}-2$, both as directly acting drugs or as add-on. Multiple pathways, as reviewed, could be targeted with these drugs, leading on to disruption of the egress of COVID-19. Since the effect of PPIs on the endosomal and lysosomal $\mathrm{pH}$ are almost similar to that of lysosomotropic agents such as hydroxychloroquine which have been considered for trials against SARS-CoV-2, PPIs should also be considered for randomized controlled and observational outcome registry based studies to understand their efficacy, dose and duration required for the treatment of COVID-19.

To conclude, the existing evidences support the notion of re-purposing PPIs against SARS-CoV-2.

\section{Author contributions}

A. R. and S.S. conceived and planned the manuscript; A.R. performed the literature review for antiinflammatory, anti-fibrotic, vacuolar ATPase and COPD related information and wrote the related sections of the manuscript; S.S. performed the literature review for ESCRT pathway and ACE2, linking it to the gastrointestinal symptoms of COVID-19 and wrote the related sections of the manuscript; B.S. integrated the manuscript; all authors performed the critical revision of the manuscript, read and approved the final version of the manuscript. 


\section{Declaration of Conflicts of Interest}

The authors declare that they have no conflict of interest.

\section{Funding}

This study has not received any specific grant from any funding agency in the public, commercial or notfor-profit sectors.

\section{References}

Aragão, D.S., Cunha, T.S., Arita, D.Y., Andrade, M.C.C., Fernandes, A.B., Watanabe, I.K.M., Mortara, R.A. \& Casarini, D.E. (2011). Purification and characterization of angiotensin converting enzyme 2 (ACE2) from murine model of mesangial cell in culture.International Journal of Biological Macromolecules , 49(1), 79-84.

Bayer, N., Schober, D., Prchla, E., Murphy, R.F., Blaas, D. \& Fuchs, R. (1998). Effect of bafilomycin A1 and nocodazole on endocytic transport in HeLa cells: implications for viral uncoating and infection.Journal of Virology , 72(12), 9645-9655.

Becker, J.C., Grosser, N., Waltke, C., Schulz, S., Erdmann, K., Domschke, W., Schröder, H. \& Pohle, T. (2006). Beyond gastric acid reduction: proton pump inhibitors induce heme oxygenase-1 in gastric and endothelial cells. Biochemical and Biophysical Research Communications , 345(3), 1014-1021.

Belouzard, S., Millet, J.K., Licitra, B.N. \& Whittaker, G.R. (2012). Mechanisms of coronavirus cell entry mediated by the viral spike protein. Viruses , 4(6), 1011-1033.

Brecher, M., Schornberg, K.L., Delos, S.E., Fusco, M.L., Saphire, E.O. \& White, J.M. (2012). Cathepsin cleavage potentiates the Ebola virus glycoprotein to undergo a subsequent fusion-relevant conformational change. Journal of Virology , 86(1), 364-372.

Casanova, C., Baudet, J.S., del Valle Velasco, M., Martin, J.M., Aguirre-Jaime, A., de Torres, J.P., Pablo de Torres, J. \& Celli, B.R. (2004). Increased gastro-oesophageal reflux disease in patients with severe COPD. The European Respiratory Journal , 23(6), 841-845.

CDC (2020). Coronavirus Disease 2019 (COVID-19) . [Online] 11 February 2020. Centers for Disease Control and Prevention. Available from: https://www.cdc.gov/coronavirus/2019-ncov/hcp/clinical-tips-forhealthcare-providers.html. [Accessed: 27 May 2020].

Chen, H.-W., Cheng, J.X., Liu, M.-T., King, K., Peng, J.-Y., Zhang, X.-Q., Wang, C.-H., Shresta, S., Schooley, R.T. \& Liu, Y.-T. (2013). Inhibitory and combinatorial effect of diphyllin, a v-ATPase blocker, on influenza viruses. Antiviral Research , 99(3), 371-382.

Chua, H.-H., Lee, H.-H., Chang, S.-S., Lu, C.-C., Yeh, T.-H., Hsu, T.-Y., Cheng, T.-H., Cheng, J.-T., Chen, M.-R. \& Tsai, C.-H. (2007). Role of the TSG101 gene in Epstein-Barr virus late gene transcription. Journal of Virology , 81(5), 2459-2471.

Cj, H., Yt, C., Zs, F., Ws, C. \& Hw, C. (2018). Antiviral efficacy of nanoparticulate vacuolar ATPase inhibitors against influenza virus infection. International Journal of Nanomedicine . 13. p.pp. 8579-8593.

Wrapp, D., Wang, N., Corbett, K.S., Goldsmith, J.A. \& Hsieh, C.L. (2020). Cryo-EM Structure of the 2019-nCoV Spike in the Prefusion Conformation. Science, 367, 1260-1263.

De Milito, A. \& Fais, S. (2005). Proton pump inhibitors may reduce tumour resistance. Expert Opinion on Pharmacotherapy , 6(7), 1049-1054.

De Milito, A., Iessi, E., Logozzi, M., Lozupone, F., Spada, M., Marino, M.L., Federici, C., Perdicchio, M., Matarrese, P., Lugini, L., Nilsson, A. \& Fais, S. (2007). Proton pump inhibitors induce apoptosis of human B-cell tumors through a caspase-independent mechanism involving reactive oxygen species. Cancer Research , 67(11), 5408-5417. 
de Duve, C., de Barsy, T., Poole, B., Trouet, A., Tulkens, P. \& Van Hoof, F. (1974). Commentary. Lysosomotropic agents. Biochemical Pharmacology , 23(18), 2495-2531.

Farrell, C.P., Morgan, M., Rudolph, D.S., Hwang, A., Albert, N.E., Valenzano, M.C., Wang, X., Mercogliano, G. \& Mullin, J.M. (2011). Proton Pump Inhibitors Interfere With Zinc Absorption and Zinc Body Stores. Gastroenterology Research , 4(6), 243-251.

Freedberg, D.E., Conigliaro, J., Wang, T.C., Tracey, K.J., Callahan, M.V., Abrams, J.A., Sobieszczyk, M.E., Markowitz, D.D., Gupta, A., O'Donnell, M.R., Li, J., Tuveson, D.A., Jin, Z., Turner, W.C. \& Landry, D.W. (2020). Famotidine Use is Associated with Improved Clinical Outcomes in Hospitalized COVID-19 Patients: A Propensity Score Matched Retrospective Cohort Study. Gastroenterology . [Online]. Available from: https://www.gastrojournal.org/article/S0016-5085(20)34706-5/abstract. [Accessed: 27 May 2020].

Geleris, J., Sun, Y., Platt, J., Zucker, J., Baldwin, M., Hripcsak, G., Labella, A., Manson, D., Kubin, C., Barr, R.G., Sobieszczyk, M.E. \& Schluger, N.W. (2020). Observational Study of Hydroxychloroquine in Hospitalized Patients with Covid-19. The New England Journal of Medicine. DOI: 10.1056/NEJMoa2012410.

George, S.N., Garcha, D.S., Mackay, A.J., Patel, A.R.C., Singh, R., Sapsford, R.J., Donaldson, G.C. \& Wedzicha, J.A. (2014). Human rhinovirus infection during naturally occurring COPD exacerbations. The European Respiratory Journal , 44(1), 87-96.

Ghebre, Y.T. \& Raghu, G. (2016). Idiopathic Pulmonary Fibrosis: Novel Concepts of Proton Pump Inhibitors as Antifibrotic Drugs. American Journal of Respiratory and Critical Care Medicine, 193(12), $1345-1352$.

Irie, T., Licata, J.M., McGettigan, J.P., Schnell, M.J. \& Harty, R.N. (2004). Budding of PPxY-Containing Rhabdoviruses Is Not Dependent on Host Proteins TGS101 and VPS4A. Journal of Virology , 78(6), 26572665 .

Kaur, G., Kaur, M. \& Silakari, O. (2014). Benzimidazoles: an ideal privileged drug scaffold for the design of multitargeted anti-inflammatory ligands. Mini Reviews in Medicinal Chemistry , 14(9), 747-767.

Kountouras, J., Boura, P. \& Lygidakis, N.J. (2000). Omeprazole and regulation of cytokine profile in Helicobacter pylori-infected patients with duodenal ulcer disease. Hepato-Gastroenterology . 47 (35). p.pp. 1301-1304.

Lee, Y.-Y., Jeon, H.-K., Hong, J.E., Cho, Y.J., Ryu, J.Y., Choi, J.-J., Lee, S.H., Yoon, G., Kim, W.Y., Do, I.-G., Kim, M.K., Kim, T.-J., Choi, C.H., Lee, J.-W., Bae, D.-S. \& Kim, B.-G. (2015). Proton pump inhibitors enhance the effects of cytotoxic agents in chemoresistant epithelial ovarian carcinoma. Oncotarget , 6(33), 35040-35050.

Lin, C.-W., Tsai, F.-J., Wan, L., Lai, C.-C., Lin, K.-H., Hsieh, T.-H., Shiu, S.-Y. \& Li, J.-Y. (2005). Binding interaction of SARS coronavirus $3 \mathrm{CL}$ (pro) protease with vacuolar-H+ ATPase G1 subunit. FEBS letters, 579(27), 6089-6094.

Liu, C., Wu, C., Zheng, X., Zeng, F., Liu, J., Wang, P., Zeng, F., Yuan, L., Zhu, F., Gan, X. \& Huang, Y. (2020). Clinical features and multidisciplinary treatment outcome of COVID-19 pneumonia: A report of three cases. Journal of the Formosan Medical Association. DOI: 10.1016/j.jfma.2020.04.008.

Long, J., Wright, E., Molesti, E., Temperton, N. \& Barclay, W. (2015). Antiviral therapies against Ebola and other emerging viral diseases using existing medicines that block virus entry. F1000Research, 4, 30.

Lu, R., Zhao, X., Li, J., Niu, P., Yang, B., Wu, H., Wang, W., Song, H., Huang, B., Zhu, N., Bi, Y., Ma, X., Zhan, F., Wang, L., Hu, T., Zhou, H., Hu, Z., Zhou, W., Zhao, L., Chen, J., Meng, Y., Wang, J., Lin, Y., Yuan, J., Xie, Z., Ma, J., Liu, W.J., Wang, D., Xu, W., Holmes, E.C., Gao, G.F., Wu, G., Chen, W., Shi, W. \& Tan, W. (2020). Genomic characterisation and epidemiology of 2019 novel coronavirus: implications for virus origins and receptor binding. The Lancet , 395(10224), 565-574. 
Marshansky, V. \& Futai, M. (2008). The V-type H+-ATPase in vesicular trafficking: targeting, regulation and function. Current Opinion in Cell Biology, 20(4), 415-426.

Mazzon, M. \& Marsh, M. (2019). Targeting viral entry as a strategy for broad-spectrum antivirals. F1000Research, 8, F1000.

Nelson, N. (1991). Structure and pharmacology of the proton-ATPases. Trends in Pharmacological Sciences , 12, 71-75.

Ohara, T. \& Arakawa, T. (1999). Lansoprazole decreases peripheral blood monocytes and intercellular adhesion molecule-1-positive mononuclear cells. Digestive Diseases and Sciences , 44(8), 1710-1715.

Patten, G.S. \& Abeywardena, M.Y. (2017). Effects of Antihypertensive Agents on Intestinal Contractility in the Spontaneously Hypertensive Rat: Angiotensin Receptor System Downregulation by Losartan. The Journal of Pharmacology and Experimental Therapeutics , 360(2), 260-266.

Prentice, E., McAuliffe, J., Lu, X., Subbarao, K. \& Denison, M.R. (2004). Identification and characterization of severe acute respiratory syndrome coronavirus replicase proteins. Journal of Virology, 78(18), 9977-9986.

PubChem (1999). Method of using $(\mathrm{H}+/ \mathrm{K}+)$ ATPase inhibitors as antiviral agents. [Online] Available from: https://pubchem.ncbi.nlm.nih.gov/patent/US5945425. [Accessed: 27 May 2020].

Reeves, P.G. \& O'Dell, B.L. (1986). Effects of dietary zinc deprivation on the activity of angiotensinconverting enzyme in serum of rats and guinea pigs. The Journal of Nutrition , 116(1), 128-134.

Sabolić, I., Brown, D., Verbavatz, J.M. \& Kleinman, J. (1994). H(+)-ATPases of renal cortical and medullary endosomes are differentially sensitive to Sch-28080 and omeprazole. The American Journal of Physiology , 266, F868-F877.

Sachs, G., Prinz, C., Loo, D., Bamberg, K., Besancon, M. \& Shin, J.M. (1994). Gastric acid secretion: activation and inhibition. The Yale Journal of Biology and Medicine , 67(3-4), 81-95.

Saliba, K.J. \& Kirk, K. (1999). pH regulation in the intracellular malaria parasite, Plasmodium falciparum. $\mathrm{H}(+)$ extrusion via a V-type $\mathrm{H}(+)$-ATPase. The Journal of Biological Chemistry , 274(47), 33213-33219.

Sasaki, T., Nakayama, K., Yasuda, H. \& Yamaya, M. (2011). A new strategy with proton pump inhibitors for the prevention of acute exacerbations in COPD. Therapeutic Advances in Respiratory Disease , 5(2), 91-103.

Sasaki, T., Nakayama, K., Yasuda, H., Yoshida, M., Asamura, T., Ohrui, T., Arai, H., Araya, J., Kuwano, K. \& Yamaya, M. (2009). A randomized, single-blind study of lansoprazole for the prevention of exacerbations of chronic obstructive pulmonary disease in older patients. Journal of the American Geriatrics Society , 57(8), $1453-1457$.

Sasaki, T., Yamaya, M., Yasuda, H., Inoue, D., Yamada, M., Kubo, H., Nishimura, H. \& Sasaki, H. (2005). The proton pump inhibitor lansoprazole inhibits rhinovirus infection in cultured human tracheal epithelial cells. European Journal of Pharmacology , 509(2-3), 201-210.

Simmons, G., Gosalia, D.N., Rennekamp, A.J., Reeves, J.D., Diamond, S.L. \& Bates, P. (2005). Inhibitors of cathepsin L prevent severe acute respiratory syndrome coronavirus entry. Proceedings of the National Academy of Sciences of the United States of America . 102 (33). p.pp. 11876-11881.

Sobhy, H. (2016). A Review of Functional Motifs Utilized by Viruses.Proteomes . 4(1), 3.

Strickland, M., Ehrlich, L.S., Watanabe, S., Khan, M., Strub, M.-P., Luan, C.-H., Powell, M.D., Leis, J., Tjandra, N. \& Carter, C.A. (2017). Tsg101 chaperone function revealed by HIV-1 assembly inhibitors. Nature Communications , 8(1), 1391.

Su, V.Y.-F., Liao, H.-F., Perng, D.-W., Chou, Y.-C., Hsu, C.-C., Chou, C.-L., Chang, Y.-L., Yen, J.-C., Chen, T.-J. \& Chou, T.-C. (2018). Proton pump inhibitors use is associated with a lower risk of acute 
exacerbation and mortality in patients with coexistent COPD and GERD.International Journal of Chronic Obstructive Pulmonary Disease, 13, 2907-2915.

Tabata, K., Arimoto, M., Arakawa, M., Nara, A., Saito, K., Omori, H., Arai, A., Ishikawa, T., Konishi, E., Suzuki, R., Matsuura, Y. \& Morita, E. (2016). Unique Requirement for ESCRT Factors in Flavivirus Particle Formation on the Endoplasmic Reticulum. Cell Reports , 16(9), 2339-2347.

Turner, A.J., Tipnis, S.R., Guy, J.L., Rice, G. \& Hooper, N.M. (2002). ACEH/ACE2 is a novel mammalian metallocarboxypeptidase and a homologue of angiotensin-converting enzyme insensitive to ACE inhibitors. Canadian Journal of Physiology and Pharmacology , 80(4), 346-353.

Udelnow, A., Kreyes, A., Ellinger, S., Landfester, K., Walther, P., Klapperstueck, T., Wohlrab, J., HenneBruns, D., Knippschild, U. \& Würl, P. (2011). Omeprazole inhibits proliferation and modulates autophagy in pancreatic cancer cells. PloS One, 6(5), e20143.

Vanfleteren, L.E.G.W., Spruit, M.A., Wouters, E.F.M. \& Franssen, F.M.E. (2016). Management of chronic obstructive pulmonary disease beyond the lungs. The Lancet. Respiratory Medicine , 4(11), 911-924.

Votteler, J. \& Sundquist, W.I. (2013). Virus budding and the ESCRT pathway. Cell Host \& Microbe, 14(3), 232-241.

Wang, H., Yang, P., Liu, K., Guo, F., Zhang, Y., Zhang, G. \& Jiang, C. (2008). SARS coronavirus entry into host cells through a novel clathrin- and caveolae-independent endocytic pathway. Cell Research , 18(2), 290-301.

Watanabe, S.M., Ehrlich, L.S., Strickland, M., Li, X., Soloveva, V., Goff, A.J., Stauft, C.B., BhaduriMcIntosh, S., Tjandra, N. \& Carter, C. (2020). Selective Targeting of Virus Replication by Proton Pump Inhibitors. Scientific Reports, 10(1), 4003.

Watanabe, T., Suzuki, T. \& Suzuki, Y. (1990). Ouabain-sensitive K(+)-ATPase in epithelial cells from guinea pig distal colon. The American Journal of Physiology, 258(4 Pt 1), G506-G511.

WHO (2020). R\&D Blueprint and COVID-19. [Online] Available from: https://www.who.int/teams/blueprint/covid-19. [Accessed: 27 May 2020].

Xiao, F., Tang, M., Zheng, X., Liu, Y., Li, X. \& Shan, H. (2020). Evidence for Gastrointestinal Infection of SARS-CoV-2.Gastroenterology . 158 (6). p.pp. 1831-1833.e3.

Yang, X., Li, Y., Sun, Y., Zhang, M., Guo, C., Mirza, I.A. \& Li, Y.-Q. (2018). Vonoprazan: A Novel and Potent Alternative in the Treatment of Acid-Related Diseases. Digestive Diseases and Sciences , 63(2), $302-311$.

Ye, Q., Wang, B. \& Mao, J. (2020). The pathogenesis and treatment of the 'Cytokine Storm' in COVID-19. The Journal of Infection , 80(6), 607-613

Yu, L.-Y., Sun, L.-N., Zhang, X.-H., Li, Y.-Q., Yu, L., Yuan, Z.-Q.-Y., Meng, L., Zhang, H.-W. \& Wang, Y.-Q. (2017). A Review of the Novel Application and Potential Adverse Effects of Proton Pump Inhibitors.Advances in Therapy , 34(5), 1070-1086.

Clinicaltrial.gov. (2020). A Randomized, Open-label Study to Evaluate the Efficacy and Safety of Pirfenidone in Patients With Severe and Critical Novel Coronavirus Infection. Available from: https://clinicaltrials.gov/ct2/show/NCT04282902. [Accessed: 27 May 2020].

Ziebuhr, J., Snijder, E.J. \& Gorbalenya, A.E. (2000). Virus-encoded proteinases and proteolytic processing in the Nidovirales. The Journal of General Virology, 81(Pt 4), 853-879. 